University of Nebraska - Lincoln

DigitalCommons@University of Nebraska - Lincoln

Biological Systems Engineering: Papers and

Publications

Biological Systems Engineering

2008

\title{
Rule-based Mamdani-type fuzzy modeling of skin permeability
}

Deepak R. Keshwani

University of Nebraska-Lincoln, dkeshwani2@unl.edu

David D. Jones

University of Nebraska-Lincoln, david.jones@unl.edu

George E. Meyer

University of Nebraska-Lincoln, gmeyer1@unl.edu

Rhonda M. Brand

Feinberg School of Medicine at Northwestern University

Follow this and additional works at: https://digitalcommons.unl.edu/biosysengfacpub

Part of the Biological Engineering Commons

Keshwani, Deepak R.; Jones, David D.; Meyer, George E.; and Brand, Rhonda M., "Rule-based Mamdani-type fuzzy modeling of skin permeability" (2008). Biological Systems Engineering: Papers and Publications. 80. https://digitalcommons.unl.edu/biosysengfacpub/80

This Article is brought to you for free and open access by the Biological Systems Engineering at DigitalCommons@University of Nebraska - Lincoln. It has been accepted for inclusion in Biological Systems Engineering: Papers and Publications by an authorized administrator of DigitalCommons@University of Nebraska Lincoln. 


\title{
Rule-based Mamdani-type fuzzy modeling of skin permeability
}

\author{
Deepak R. Keshwani, ${ }^{1}$ David D. Jones, ${ }^{2}$ George E. Meyer, ${ }^{2}$ Rhonda M. Brand ${ }^{3}$ \\ ${ }^{1}$ Department of Biological \& Agricultural Engineering, \\ North Carolina State University, Raleigh, NC 27695 \\ 2 Department of Biological Systems Engineering, University of Nebraska-Lincoln, \\ 215 L. W. Chase Hall, East Campus, Lincoln, NE 68583-0726 \\ ${ }^{3}$ Department of Internal Medicine, Evanston Northwestern Healthcare, \\ Feinberg School of Medicine, Evanston, IL 60201 \\ Corresponding author - D. D. Jones, tel 402 472-6716, fax 402 472-6338, email djones1@unl.edu
}

\begin{abstract}
Two Mamdani type fuzzy models (three inputs-one output and two inputs-one output) were developed to predict the permeability of compounds through human skin. The models were derived from multiple data sources including laboratory data, published data bases, published statistical models, and expert opinion. The inputs to the model include information about the compound (molecular weight and octonal- $\mathrm{H}_{2} \mathrm{O}$ partition coefficient) and the application temperature. One model included all three parameters as inputs and the other model only included information about the compound. The values for mole molecular weight ranged from 30 to $600 \mathrm{Da}$. The values for the $\log$ of the octonal- $\mathrm{H}_{2} \mathrm{O}$ partition coefficient ranged from -3.1 to 4.34 . The values for the application temperature ranged from 22 to $398 \mathrm{C}$. The predicted values of the log of permeability coefficient ranged from -5.5 to 0.08. Each model was a collection of rules that express the relationship of each input to the permeability of the compound through human skin. The quality of the model was determined by comparing predicted and actual fuzzy classification and defuzzification of the predicted outputs to get crisp values for correlating estimates with published values. A modified form of the Hamming distance measure is proposed to compare predicted and actual fuzzy classification. An entropy measure is used to describe the ambiguity associated with the predicted fuzzy outputs. The three input model predicted over $70 \%$ of the test data within one-half of a fuzzy class of the published data. The two input model predicted over $40 \%$ of the test data within one-half of a fuzzy class of the published data. Comparison of the models show that the three input model exhibited less entropy than the two input model.
\end{abstract}

Keywords: Mamdani fuzzy modeling, Hamming distance, skin permeability

\section{Introduction}

Determination of skin permeability is an important issue in the area of transdermal drug delivery and environmental toxicity. Transdermal delivery offers a less invasive means to administer drugs. In addition the concentration of the drugs can be maintained at a steady state. Identifying a compound's potential to be toxic via a transdermal route is critical for certain high-risk occupations such as chemical manufacturers and painters.

In the area of skin permeability, a common modeling approach is to develop empirical models from experimentally derived databases $[7,9,13,14]$. However, skin permeability databases are typically small in size and numerous inconsistencies exist within them. Vecchia and Bunge provide a fully validated skin permeability database where each data point met a set of defined criteria for inclusion [17]. Despite enforcing the validation criteria, the size of the database remains small and the range of the predictors is limited. Analytical approaches have been proposed by Edwards and Langer [2] to model skin permeability. However, with this approach, assumptions are made on the behavior of the system. These assumptions are difficult to validate and the resulting description of the system is often over simplified. The functional nature of the skin as a barrier is complex. This complexity results in uncertainty that cannot exclusively be described by random measures. Hence, predicting skin permeability can be deemed an ambiguous endeavor and fuzzy modeling provides a mean to account for this ambiguity. Estimating the skin permeability coefficients of compounds is vital to determining potential for toxic exposure and transdermal drug delivery.

Pannier et al. [11] and Keshwani et al. [6] have shown that rule-based fuzzy modeling of skin permeability is a 
promising approach. However, the rules for these models were strictly data driven and examination of the results revealed inconsistencies that can be attributed to sparse data in some regions. This paper presents a Mamdani fuzzy modeling scheme where rules are derived from multiple knowledge sources such as previously published databases and models, existing literature, intuition and solicitation of expert opinion to verify the gathered information.

The output or consequence of a Mamdani-type model is represented by a fuzzy set. To assess model performance, a crisp estimate of the consequence is usually made by defuzzification methods such as the centroid, weighted average, maximum membership principle and mean membership principle [15]. The crisp values can be compared to the actual values from the data set and a correlation coefficient can be determined. Depending on the shape of the output fuzzy set, defuzzification methods do not effectively characterize the output with the corresponding ambiguity associated with the prediction. The nature of the ambiguity in the prediction might be of interest to researchers in the area of skin permeability. An alternative strategy could be implemented such that the actual values of the output infer an ordinal set representing a three point fuzzy classification (low, medium and high) that could be compared to the actual fuzzy classification using distance measures. In addition, the ambiguity associated with the predicted fuzzy sets can be quantified by calculating entropy [4].
The purpose of this study was to develop generalized rule based fuzzy models from multiple knowledge sources to predict skin permeability and subsequently test its performance by comparing defuzzified outputs to actual values from test data and comparing predicted and actual fuzzy classifications. The overall approach followed in this study is illustrated in Figure 1. The process begins with knowledge acquisition, continues to model building and then finally testing the model performance. In the context of skin permeability, this approach is not common in that it combines information from multiple sources for model development. In the context of fuzzy modeling, the proposed approach of converting the predicted fuzzy output and the actual crisp value into fuzzy classification sets is not well defined in literature.

\section{Theory}

\subsection{Mamdani-type fuzzy modeling}

As the complexity of a system increases, the utility of fuzzy logic as a modeling tool increases. For very complex systems, few numerical data may exist and only ambiguous and imprecise information and knowledge is available. Oduguwa et al. [10] recognized and attempted to capture qualitative aspects of the engineering design process.

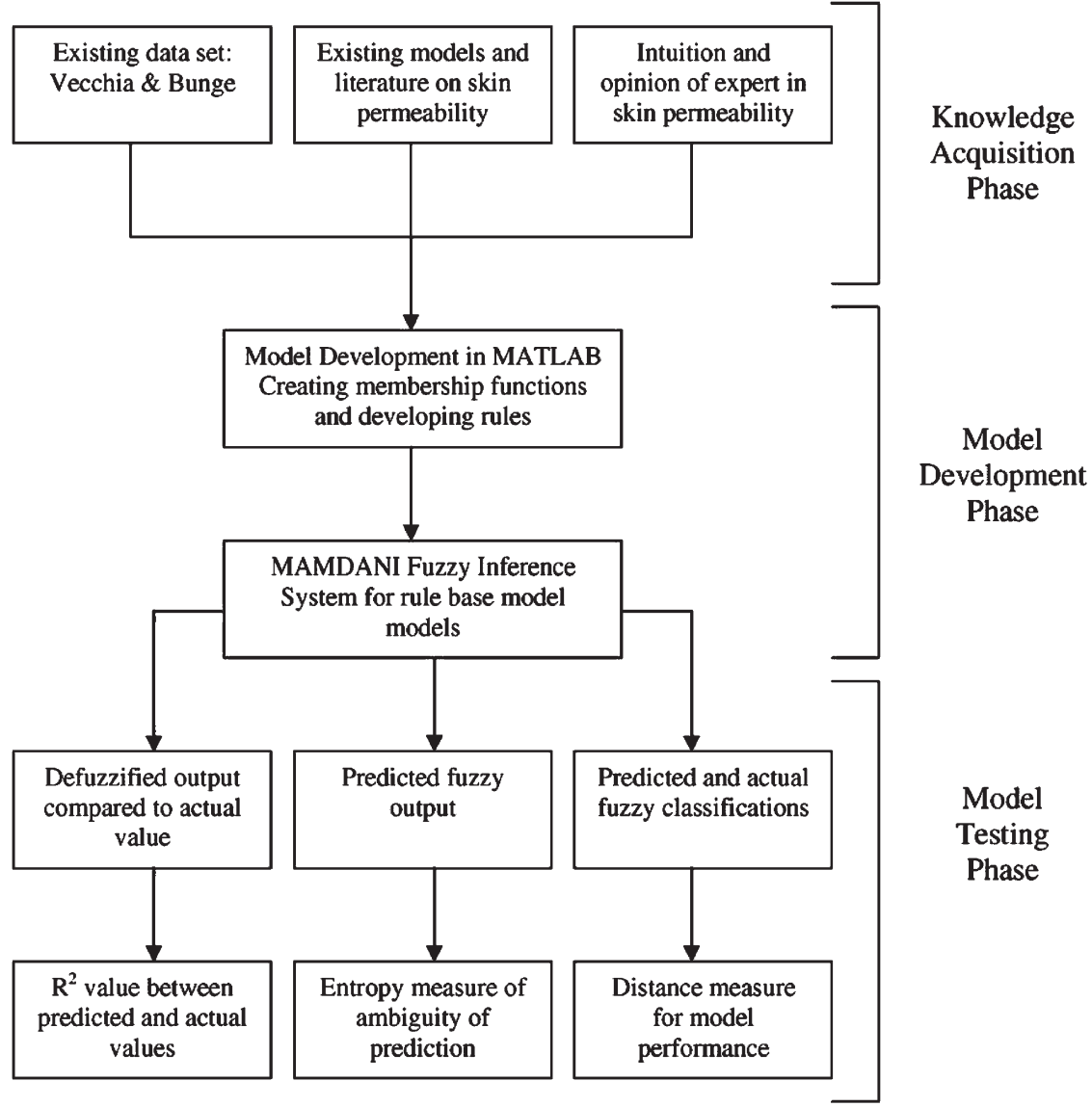

Figure 1. Overall approach to develop skin permeability Mamdani models. 


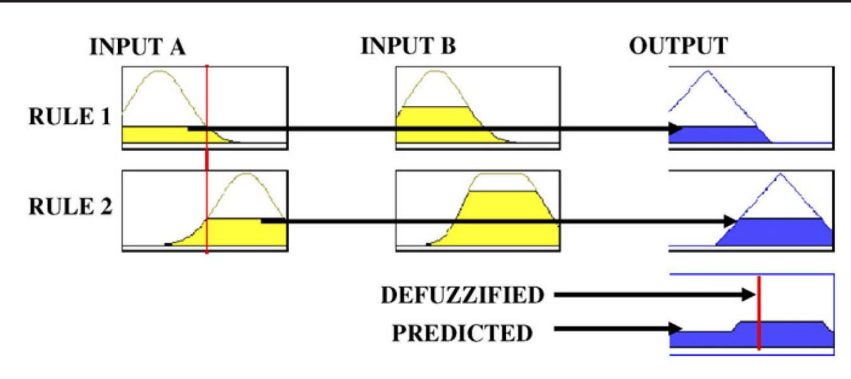

Figure 2. Example of a Mamdani type fuzzy inference system.

Fuzzy logic allows approximate interpolation between input and output situations [15]. Two main types of fuzzy modeling schemes are the Takagi-Sugeno model and the fuzzy relational model. The Takagi-Sugeno scheme is a data driven approach where membership functions and rules are developed using a training data set. The parameters for the membership functions and rules are subsequently optimized to reduce training error. The relationship in each rule is represented by a localized linear function [1]. The final output is a weighted average of a set of crisp values. The Mamdani scheme is a type of fuzzy relational model where each rule is represented by an IFTHEN relationship. It is also called a linguistic model because both the antecedent and the consequent are fuzzy propositions [1]. The model structure is manually developed and the final model is neither trained nor optimized. The output from a Mamdani model is a fuzzy membership function based on the rules created. Since this approach is not exclusively reliant on a data set, with sufficient expertise on the system involved, a generalized model for effective future predictions can be obtained.

Consider a simple two input-one output Mamdani type fuzzy model. The rule structure is represented in Figure 2. Each row of membership functions constitutes an IFTHEN rule, also defined by the user. Depending on the values used, the input membership functions are activated to a certain degree. The contributed output from each rule reflects this degree of activation. The final output is a fuzzy set created by the superposition of individual rule actions (Figure 2).

\subsection{Defuzzification methods}

The fuzzy output is obtained from aggregating the outputs from the firing of the rules. Subsequent defuzzification methods on the fuzzy output produce a crisp value. Two common techniques for defuzzification are the maxima methods and area-based methods, which are briefly explained. Several such methods are explained by Ross [15].

\subsubsection{Maxima methods}

The maxima methods identify the locations where maximum membership occurs. Either one such point is selected as the defuzzified value (Figure $3 \mathrm{~A}$ ) or an average of all points with maximum membership is selected as the crisp value (Figure 3B). The advantages of the maxima methods are their simplicity and speed [12]. The major disadvantage is loss of information as only rules of maximum activation are considered.
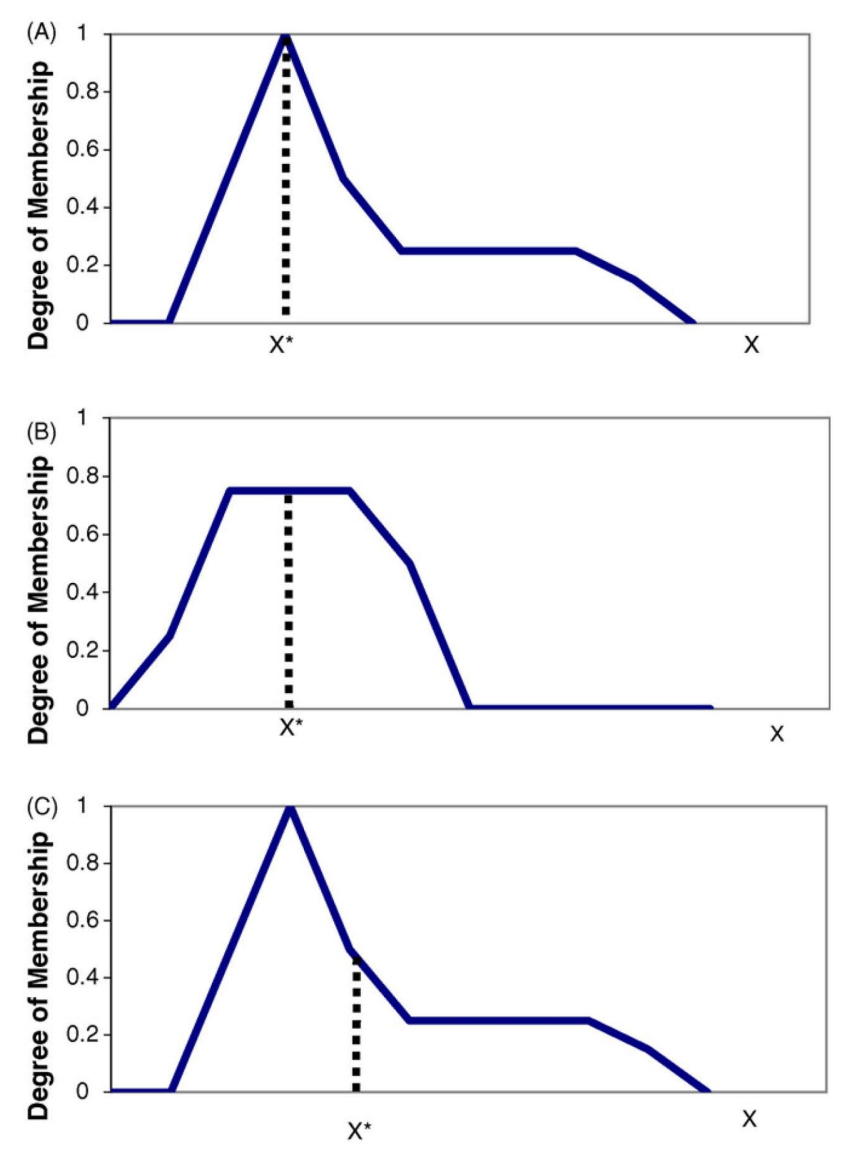

Figure 3. Different defuzzification methods: (A) max-membership principle; (B) mean-max-membership principle; (C) centroid principle. Note: $x^{*}$ is the defuzzified value.

\subsubsection{Area-based methods}

A popular area-based defuzzification procedure is the centroid method. As the term implies, the point of the output membership function that splits the area in half is selected as the crisp value (Figure 3c). This method however does not work when the output membership function has nonconvex properties.

Depending on the shape of the membership function of the output, defuzzification routines may not produce effective values for the predicted output. For example, in Figure $4 \mathrm{~A}$, the predicted output indicates a high degree of ambiguity. However, the defuzzified value using the mean-max membership principle that does not convey the ambiguity. The centroid method has drawbacks when the output membership function is non-convex (Figure 4B). The defuzzified value is at a point that has low membership. In an effort to compensate for these drawbacks, an alternative approach to model validation is proposed that uses a distance measure to compare actual and predicted fuzzy classifications consisting of three point ordinal sets.

\subsection{Distance measures between fuzzy sets}

For two fuzzy sets A and B in the same universe, the Hamming distance [16] is an ordinal measure of dissimilarity. 

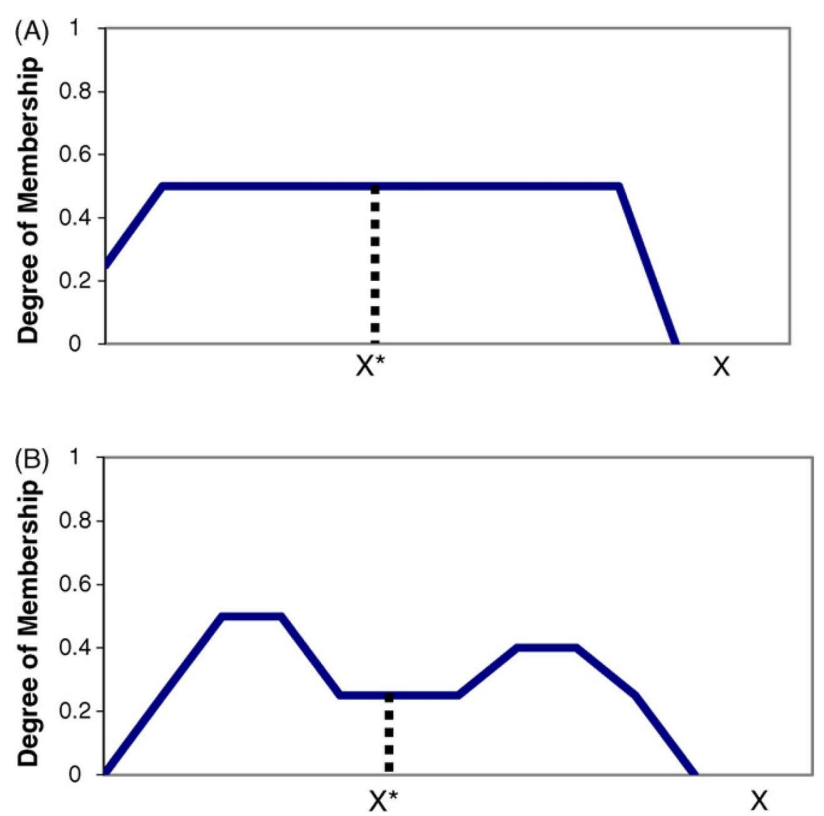

Figure 4. Problems with defuzzification methods: (A) drawback of maxima method; (B) drawback of centroid method. Note: $x^{*}$ is the defuzzified value.

The Hamming distance (HD) is defined as:

$$
\mathrm{HD}(\mathrm{A}, \mathrm{B})=\sum_{i=1}^{n}\left|\mu_{\mathrm{A}}\left(x_{i}\right)-\mu_{\mathrm{B}}\left(x_{i}\right)\right|
$$

where $n$ is the number of points that define the fuzzy sets $\mathrm{A}$ and $\mathrm{B}, \mu_{\mathrm{A}}\left(x_{i}\right)$ the membership of point $x_{i}$ in $\mathrm{A}$ and $\mu_{\mathrm{B}}\left(x_{i}\right)$ is the membership of point $x_{i}$ in $\mathrm{B}$. The Hamming distance is smaller for fuzzy sets that are more alike than those that are less similar. In comparing an actual fuzzy set to the predicted fuzzy set, a small Hamming distance is ideal. In our study, the model-testing phase involved comparison of predicted and actual fuzzy classifications (low, medium and high). For example, if the actual value was classified low and the predicted value was classified medium, then the prediction is off by one class. If the actual value was classified low and the predicted value was classified high, then the prediction is off by two classes. In this case, the classifications for actual and predicted are fuzzy (for example, 0.60 low, 0.35 medium, 0.05 high). A modified form of the Hamming distance measure is proposed in the methods section. This new measure was developed in lieu of certain drawbacks with the Hamming distance.

Consider the example classification sets in Table 1. For an actual classification set of (low $=1$, medium $=0$, high $=0$ ), the distance formula was applied to evaluate the degree of misclassifications for a number of possible predicted sets. An exact match would result in a distance of 0 . When the prediction is off by one class, the distance is 1 and when the prediction is off by two classes, the distance is 2 . The Hamming distance is also calculated in each case. From the results in Table 1, the proposed distance measure is better than the Hamming distance at distinguishing between different levels of classification. In cases $i, j, k$ and $l$, the Hamming distance (HD) gave the same value for dif-
Table 1. Sample calculations to compare the Hamming distance and the proposed modified Hamming distance

\begin{tabular}{|c|c|c|c|c|c|c|c|c|}
\hline \multirow[b]{2}{*}{ Case } & \multicolumn{3}{|c|}{$\begin{array}{l}\text { Actual fuzzy } \\
\text { classification }\end{array}$} & \multicolumn{3}{|c|}{$\begin{array}{l}\text { Predicted fuzzy } \\
\text { classification }\end{array}$} & \multirow[b]{2}{*}{$D^{\mathrm{a}}$} & \multirow[b]{2}{*}{$\mathrm{HD}^{\mathrm{b}}$} \\
\hline & Low & Medium & High & Low & Medium & High & & \\
\hline a & 1.0 & 0.0 & 0.0 & 1.0 & 0.0 & 0.0 & 0.0 & 0.0 \\
\hline $\mathrm{b}$ & 1.0 & 0.0 & 0.0 & 0.9 & 0.1 & 0.0 & 0.1 & 0.2 \\
\hline c & 1.0 & 0.0 & 0.0 & 0.8 & 0.2 & 0.0 & 0.2 & 0.4 \\
\hline $\mathrm{d}$ & 1.0 & 0.0 & 0.0 & 0.8 & 0.1 & 0.1 & 0.3 & 0.4 \\
\hline e & 1.0 & 0.0 & 0.0 & 0.6 & 0.4 & 0.0 & 0.4 & 0.8 \\
\hline $\mathrm{f}$ & 1.0 & 0.0 & 0.0 & 0.5 & 0.5 & 0.0 & 0.5 & 1.0 \\
\hline $\mathrm{g}$ & 1.0 & 0.0 & 0.0 & 0.6 & 0.2 & 0.2 & 0.6 & 0.8 \\
\hline $\mathrm{h}$ & 1.0 & 0.0 & 0.0 & 0.5 & 0.3 & 0.2 & 0.7 & 1.0 \\
\hline i & 1.0 & 0.0 & 0.0 & 0.0 & 1.0 & 0.0 & 1.0 & 2.0 \\
\hline j & 1.0 & 0.0 & 0.0 & 0.0 & 0.2 & 0.8 & 1.8 & 2.0 \\
\hline $\mathrm{k}$ & 1.0 & 0.0 & 0.0 & 0.0 & 0.1 & 0.9 & 1.9 & 2.0 \\
\hline 1 & 1.0 & 0.0 & 0.0 & 0.0 & 0.0 & 1.0 & 2.0 & 2.0 \\
\hline
\end{tabular}

a Modified Hamming distance calculated using Equation (3).

b Hamming distance calculated using Equation (1).

ferent predicted fuzzy classifications. The proposed modified Hamming distance gave different values that effectively distinguish between these cases.

\subsection{Entropy of a fuzzy set}

Entropy is a measure of fuzziness associated with a fuzzy set. The degree of fuzziness can be described in terms of a lack of distinction between a fuzzy set and its complement. For a fuzzy set A, entropy [7] is calculated as:

$$
E(\mathrm{~A})=1-\frac{1}{n} \sum_{i=1}^{n}\left|2 \mu_{\mathrm{A}}\left(x_{i}\right)-1\right|
$$

where $n$ is the number of points that define $\mathrm{A}$, and $\mu_{\mathrm{A}}\left(x_{i}\right)$ is the membership of point $x_{i}$ in A. In this study, the concept of entropy was used to quantify the ambiguity associated with the predicted fuzzy outputs. In the absence of actual values, entropy values are essentially a measure of confidence in outputs predicted by a fuzzy model.

\section{Methods}

\subsection{Knowledge acquisition phase}

A Mamdani-type fuzzy model involves developing membership functions and defining the subsequent rules. Three main knowledge sources were used to obtain information in this regard. A description of these sources and examples of information acquired from each are described below.

\subsubsection{Skin permeability database}

A fully validated database from Vecchia and Bunge [17] was used as a guide during model development. This database is one of the most comprehensive available, where 
each included case has to meet pre-define criteria to validate its inclusion. Information on octanol-water partition coefficient $\left(\log K_{\mathrm{ow}}\right)$, molecular weight $\left(M_{\mathrm{W}}\right)$, temperature $(T)$, experimental skin permeability coefficients $\left(\log K_{\mathrm{p}}\right)$ are some of the parameters included for each point in the database. $\log K_{\text {ow }}$ ranged from -3 to $5, M_{W}$ ranged from 30 to 600 , temperature ranged from 22 to $39^{\circ} \mathrm{C}$ and $\log K_{\mathrm{p}}$ ranged from -6 to 0 . The database was helpful in determining the number of membership functions needed for each parameter included in the models and their properties. For example, prior work that involved developing a data driven fuzzy model using this database indicated that 25,32 and $37^{\circ} \mathrm{C}$ were suitable position for the centers of membership functions in the temperature domain [6].

\subsubsection{Skin permeability literature and previous models}

Discussion on the theory of skin permeability and the barrier nature of the skin are provided by Flynn [3]. Published models by Potts and Guy [13,14], Moody et al. [9] and Kirchner et al. [7], Pannier et al. [11], Keshwani et al. [6] and Magnusson et al. [8] provide an understanding on the influence of certain input parameters on skin permeability and the corresponding impact on assigning membership functions. For example, a review of literature indicates that hydrophilic and lipophilic compounds may follow different pathways in penetrating the skin [14]. This information is reflected in the discontinuity between the membership functions for compounds that are hydrophilic and lipophilic (seen in Figure 7).

\subsubsection{Expert opinion}

The database, literature and models can only guide the development of preliminary membership functions and rules. For data driven fuzzy models, optimization routines modify the membership functions for a training data set. For Mamdani models, solicitation of expert opinion can be considered a pseudo-optimization step. The main information solicited from the expert was regarding the nature of the inputs and output membership functions and the subsequent rules. For example, it was suggested that the effect of molecular weight levels off at both low and high extremes. This information is reflected in the shape of the low and high membership functions on the molecular weight domain (seen in Figure 8).

\subsection{Model development phase}

Two Mamdani models were created. The inputs used in the first model were $\log K_{\mathrm{ow}^{\prime}}$ molecular weight, and temperature, and the predicted output was the skin permeability coefficient $\left(\log K_{\mathrm{p}}\right)$. The inputs in the second model were $\log K_{\text {ow' }}$ and molecular weight predicting $\log K_{\mathrm{p}}$. The fuzzy logic toolbox in MATLAB [16] was used to build the fuzzy inference systems. Based on the information collected from the various sources, membership functions were created for each input and the output and subsequent rules were developed for each model. The fuzzy inference system was then presented to the expert for suitable modifications to the membership functions and the rules.

\subsection{Proposed distance measure}

As indicated in the theory section, a modified form of the Hamming distance is proposed which enables better distinction between different levels of classification (see Table 1 ). The proposed distance measure $D(\mathrm{~A}, \mathrm{P})$ is defined as:

$$
\begin{aligned}
D(\mathrm{~A}, \mathrm{P})= & \frac{1}{4}\left\{\sum_{i=1}^{n}\left|\mu_{\mathrm{A}}\left(x_{i}\right)-\mu_{\mathrm{P}}\left(x_{i}\right)\right|\right. \\
& \left.+\sum_{i, k=1_{i \neq k}}^{n}\left[2(2|i-k|-1) \mu_{\mathrm{A}}\left(x_{i}\right) \mu_{\mathrm{P}}\left(x_{k}\right)\right]\right\}
\end{aligned}
$$

where $\mathrm{A}$ is the actual fuzzy classification, $\mathrm{P}$ the predicted fuzzy classification, $n$ the number of classes that define $\mathrm{A}$ and $\mathrm{P}, \mu_{\mathrm{A}}\left(x_{i}\right)$ is the membership of point $x_{i}$ in $\mathrm{A}$ and $\mu_{\mathrm{P}}\left(x_{k}\right)$ is the membership of point $x_{k}$ in $\mathrm{P}$.

\subsection{Model testing phase}

Test data consisting of three inputs $\left(\log K_{\mathrm{ow}^{\prime}}, M_{\mathrm{W}^{\prime}}, T\right)$ and two inputs $\left(\log K_{\mathrm{ow}^{\prime}}, M_{\mathrm{W}}\right)$ was obtained from the Vecchia and Bunge database [17]. The models were tested in two ways.

\subsubsection{Comparing fuzzy classifications}

The three output membership functions created in both models are categorized as low, medium and high. The actual value from the test data was evaluated using the parameters of these membership functions to produce a fuzzy set represented by three points (Figure 5). This fuzzy set represents the degree of belongingness $(\mu)$ to each of the three categories (low, medium and high). The predicted
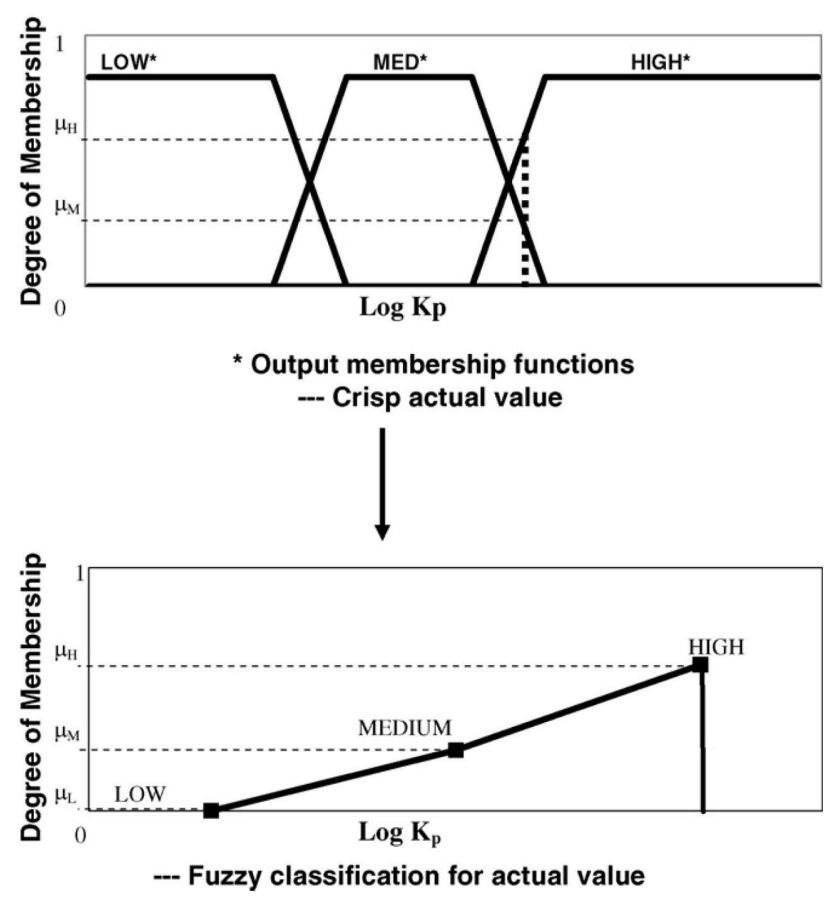

Figure 5. Obtaining fuzzy classification set for actual value. 

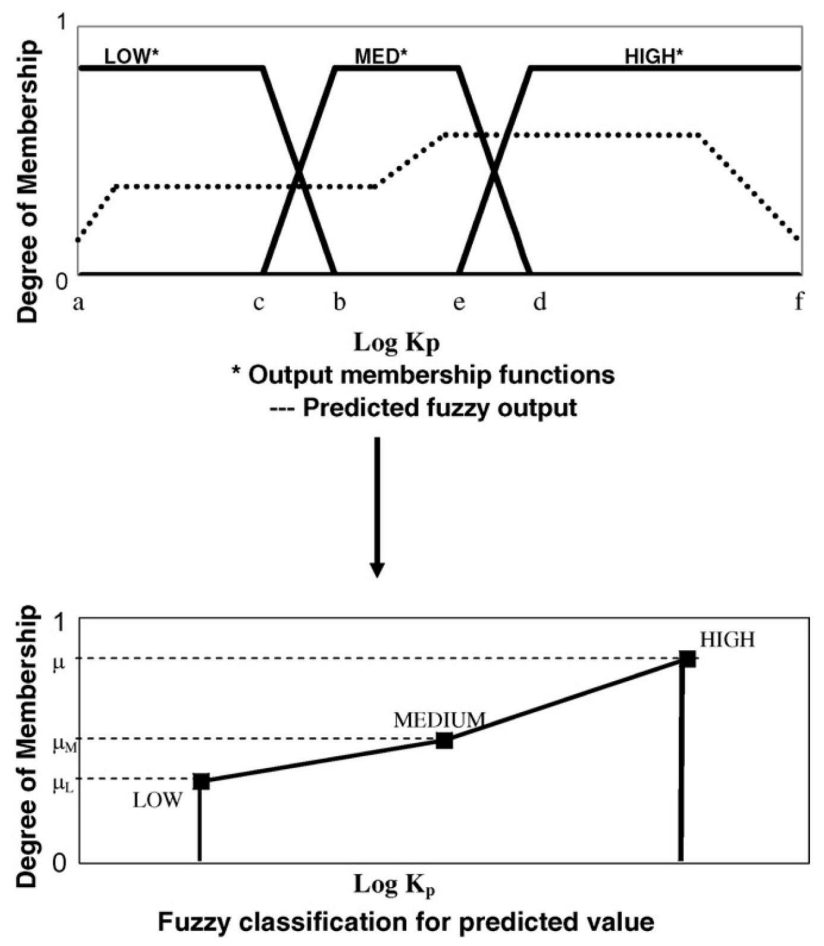

Figure 6. Obtaining fuzzy classification set for predicted fuzzy output.

output from the Mamdani model is a fuzzy set represented by 101 points. Based on the relative contributions from each output membership function (high, medium and low), the predicted fuzzy set of 101 points was reduced to a fuzzy set of three points (Figure 6). The relative contributions from each output membership function were estimated by integrating the predicted fuzzy set over the range of the membership function. Equations (4)-(6) were used to develop the predicted fuzzy classification:

$$
\begin{aligned}
& \mu_{\mathrm{L}}(\mathrm{P})=\frac{\sum_{i=a}^{b} \mu_{i}(\mathrm{P})}{\sum_{i=a}^{f} \mu_{i}(\mathrm{P})} \\
& \mu_{\mathrm{M}}(\mathrm{P})=\frac{\sum_{i=c}^{d} \mu_{i}(\mathrm{P})}{\sum_{i=a}^{f} \mu_{i}(\mathrm{P})} \\
& \mu_{\mathrm{H}}(\mathrm{P})=\frac{\sum_{i=e}^{f} \mu_{i}(\mathrm{P})}{\sum_{i=a}^{f} \mu_{i}(\mathrm{P})}
\end{aligned}
$$

In the above equations, $\mu_{\mathrm{L}}(\mathrm{P}), \mu_{\mathrm{L}}(\mathrm{P})$, and $\mu_{\mathrm{L}}(\mathrm{P})$ constitute the predicted fuzzy classification, $\mu_{i}(\mathrm{P})$ is the membership of each point in the predicted fuzzy set and a-f are the ranges of the output membership functions defined in Figure 6.

For each test case, an actual fuzzy classification and a predicted fuzzy classification were obtained. The modified Hamming distance measure (3) was used to determine the similarity between the two fuzzy sets. Apart from a comparison to actual values, the ambiguity associated with each predicted value was quantified using an entropy measure (2) as defined in the theory section.

\subsubsection{Defuzzifying the predicted output}

The centroid method was used to defuzzify the output of the Mamdani models. The crisp predictions were compared to the actual values from the test data and $R^{2}$ estimate of correlation was calculated. This is a common form of comparison utilized for most modeling strategies. However, defuzzifying the output results in a loss of information regarding the ambiguity of the prediction. In the absence of actual values, the confidence in the prediction can be determined based on the degree of ambiguity.

\section{Results}

\subsection{Membership functions and rules}

The first Mamdani model was developed with three inputs (log $K_{\mathrm{ow}^{\prime}}, M_{\mathrm{W}^{\prime}}$, and temperature) to predict $\log K_{\mathrm{p}}$ as an output. The second model was developed with two inputs $\left(\log K_{\text {ow }}\right.$ and $\left.M_{\mathrm{W}}\right)$. Four membership functions were developed for $\log K_{\text {ow }}$ (Figure 7) to linguistically represent hydrophilic to highly lipophilic compounds. The range of $\log K_{\text {ow }}$ used in the model is -4 to 8 . There is a discontinuity between the hydrophilic and the lipophilic membership functions. This stems from the hypothesis that hydrophilic compounds may penetrate the skin in a manner different from lipophilic compounds [14]. Hence, there is a lack of knowledge and information available on the compounds with a $\log K_{\text {ow }}$ that occurs between the hydrophilic and lipophilic membership functions. A Mamdani modeling scheme enables representation of this lack of knowledge in the model structure.

Three membership functions were developed for molecular weight (Figure 8) representing low, medium, and high linguistic classes. The range for $M_{\mathrm{W}}$ used in the model is from 10 to 1000. The high molecular weight membership function is important as data for most existing models does not contain information for such heavy compounds.

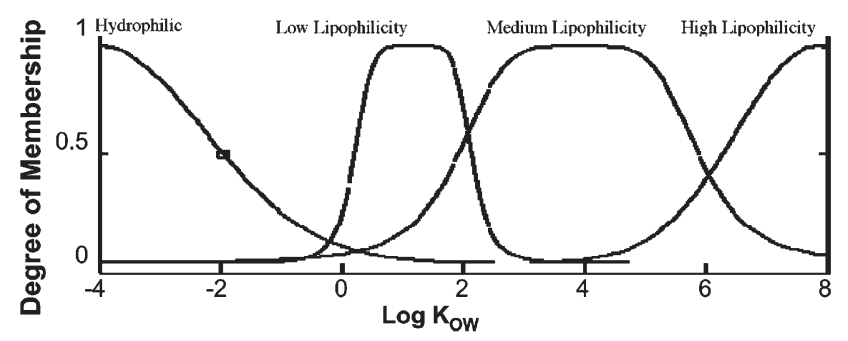

Figure 7. Membership functions for $\log K_{\text {ow }}$.

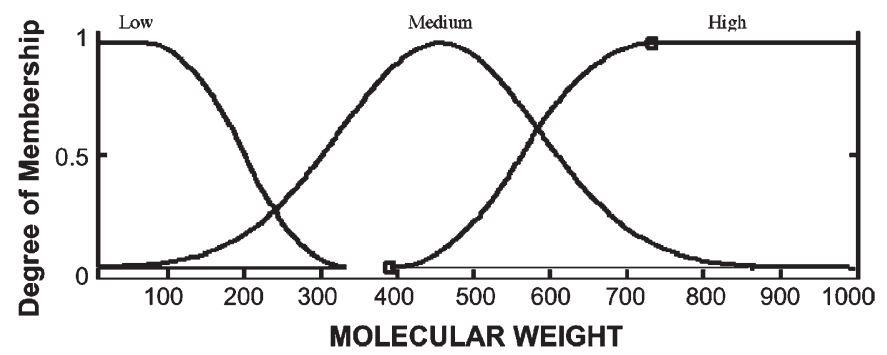

Figure 8. Membership functions for molecular weight. 


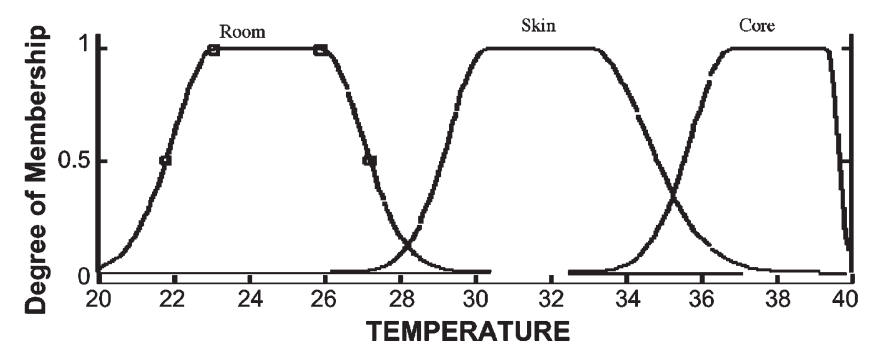

Figure 9. Membership functions for temperature.

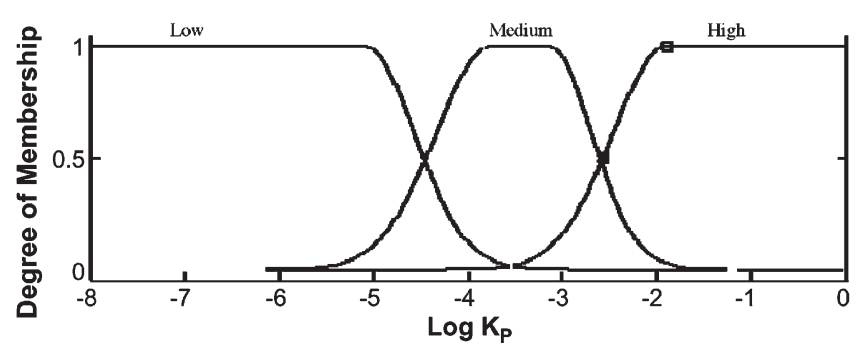

Figure 10. Membership functions for $\log K_{p}$.

Three membership functions were developed for temperature (Figure 9) representing room, skin, and core body temperature. The range for temperature was $20-40{ }^{\circ} \mathrm{C}$. Three membership functions were developed for $\log K_{\mathrm{p}}$ (Figure 10) representing low, medium, and high permeability. The range of the output was from -8 to 0 with least permeability occurring at -8 .

Based on gathered information and expert opinion, 36 rules (Table 2) were developed to map the input membership functions to the output membership functions for the three input model. Similarly, 21 rules were developed for the two input model. The two input model contains multiple rules where the same antecedents result in a different consequence. This stems from the fact that absence of temperature as an input adds more ambiguity to the prediction of $\log K_{\mathrm{p}}$. The output $\left(\log K_{\mathrm{p}}\right)$ is predicted as low, medium, or high, based on the combination of the input membership functions (Table 3).

\subsection{Comparing predicted and actual fuzzy classification}

During the testing of each model, fuzzy classifications were created for the predicted and actual values using defined

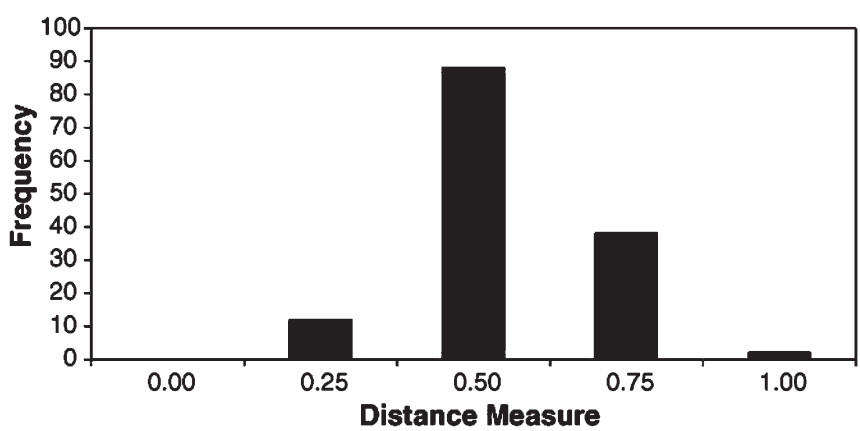

Figure 11. Calculated distance measures-using Equation (3) - for three input model test data.
Table 2 Rules developed for three input model

\begin{tabular}{|c|c|c|c|}
\hline IF $\log K_{\text {ow }}$ & $\operatorname{AND} M_{W}$ & AND $T$ & THEN $\log K_{p}$ \\
\hline Hydrophilic & Low & Room & Medium \\
\hline Hydrophilic & Low & Skin & Medium \\
\hline Hydrophilic & Low & Core & High \\
\hline Hydrophilic & Medium & Room & Low \\
\hline Hydrophilic & Medium & Skin & Medium \\
\hline Hydrophilic & Medium & Core & Medium \\
\hline Hydrophilic & High & Room & Medium \\
\hline Hydrophilic & High & Skin & Low \\
\hline Hydrophilic & High & Core & Low \\
\hline Low lipophilicity & Low & Room & Medium \\
\hline Low lipophilicity & Low & Skin & Medium \\
\hline Low lipophilicity & Low & Core & Medium \\
\hline Low lipophilicity & Medium & Room & Low \\
\hline Low lipophilicity & Medium & Skin & Low \\
\hline Low lipophilicity & Medium & Core & Medium \\
\hline Low lipophilicity & High & Room & Low \\
\hline Low lipophilicity & High & Skin & Low \\
\hline Low lipophilicity & High & Core & Low \\
\hline Medium lipophilicity & Low & Room & High \\
\hline Medium lipophilicity & Low & Skin & High \\
\hline Medium lipophilicity & Low & Core & High \\
\hline Medium lipophilicity & Medium & Room & Medium \\
\hline Medium lipophilicity & Medium & Skin & High \\
\hline Medium lipophilicity & Medium & Core & High \\
\hline Medium lipophilicity & High & Room & High \\
\hline Medium lipophilicity & High & Skin & Medium \\
\hline Medium lipophilicity & High & Core & High \\
\hline High lipophilicity & Low & Room & Low \\
\hline High lipophilicity & Low & Skin & Medium \\
\hline High lipophilicity & Low & Core & Medium \\
\hline High lipophilicity & Medium & Room & Medium \\
\hline High lipophilicity & Medium & Skin & Low \\
\hline High lipophilicity & Medium & Core & Low \\
\hline High lipophilicity & High & Room & Low \\
\hline High lipophilicity & High & Skin & Low \\
\hline High lipophilicity & High & Core & Low \\
\hline
\end{tabular}

output membership functions (Figures $6 \& 7$ ). Each fuzzy classification set was represented by three membership values: high, medium, and low. The proposed distance formula was applied in each test case and an estimate of classification was obtained. The distribution of the calculated distances for both models is provided in Figures 11 and 12. Referring back to Table 1, a distance measure of one implies that the model prediction was one fuzzy class away from the actual value. A distance measure of two implies

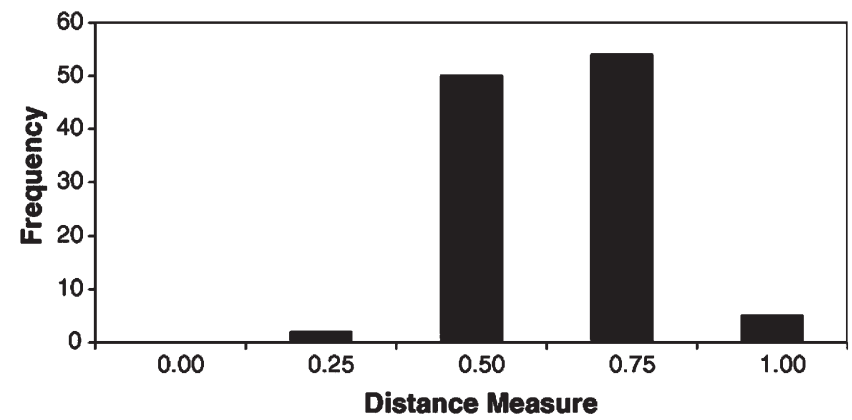

Figure 12. Calculated distance measures-using Equation (3) - for two input model test data. 


\begin{tabular}{lll} 
Table 3. Rules developed for two input model & \\
\hline IF log $K_{\text {ow }}$ & AND $M_{\mathrm{W}}$ & THEN $\log K_{\mathrm{p}}$ \\
\hline Hydrophilic & Low & Medium \\
Hydrophilic & Low & High \\
Hydrophilic & Medium & Low \\
Hydrophilic & Medium & Medium \\
Hydrophilic & High & Medium \\
Hydrophilic & High & Low \\
Low lipophilicity & Low & Medium \\
Low lipophilicity & Medium & Low \\
Low lipophilicity & Medium & Medium \\
Low lipophilicity & High & Low \\
Medium Lipophilicity & Low & High \\
Medium Lipophilicity & Medium & Medium \\
Medium Lipophilicity & Medium & High \\
Medium Lipophilicity & High & High \\
Medium Lipophilicity & High & Medium \\
High lipophilicity & Low & Low \\
High lipophilicity & Low & Medium \\
High lipophilicity & Medium & Medium \\
High lipophilicity & Medium & Low \\
High lipophilicity & High & Low \\
\hline & & \\
\hline
\end{tabular}

that the model prediction was two fuzzy classes from the actual value. Results for the three input model (Figure 13) indicate that $71 \%$ of the test data were predicted within half a fuzzy class of the actual value. For the three input model, $47 \%$ of the test data was predicted within half a fuzzy class of the actual value. In both models, all the test data was predicted within one fuzzy class of the actual value. However, the performance of the three input model does appear to be significantly better.

\subsection{Comparing predicted defuzzified values to actual values}

The fuzzy outputs from both models were defuzzified using the centroid principle (9). The crisp predictions were then compared to the actual values from the test data and estimates of RMSE and correlation were calculated. The

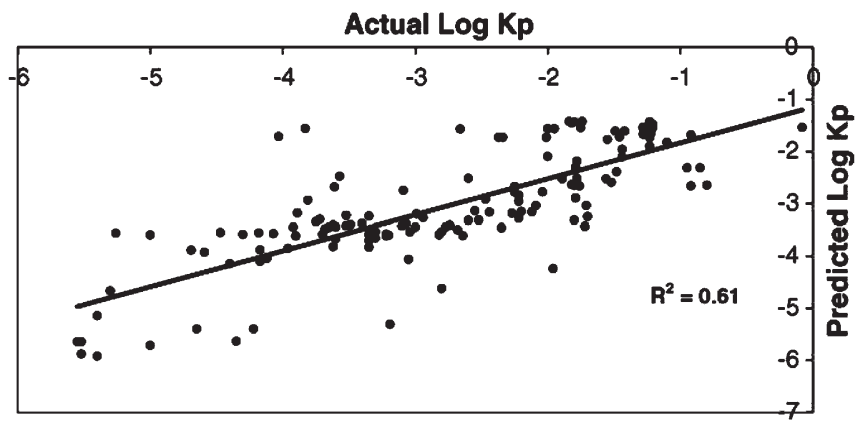

Figure 13. Actual $\log K_{p}$ vs. predicted $\log K_{p}$ for three input model test data.

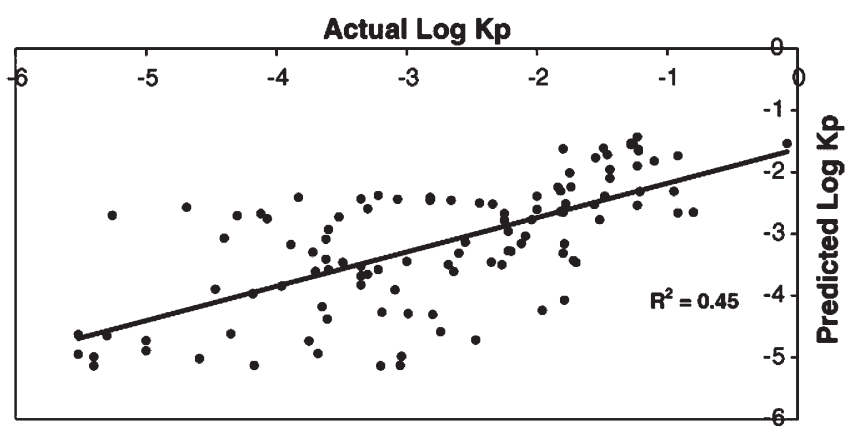

Figure 14. Actual $\log K_{\mathrm{p}}$ vs. predicted $\log K_{\mathrm{p}}$ for two input model test data.

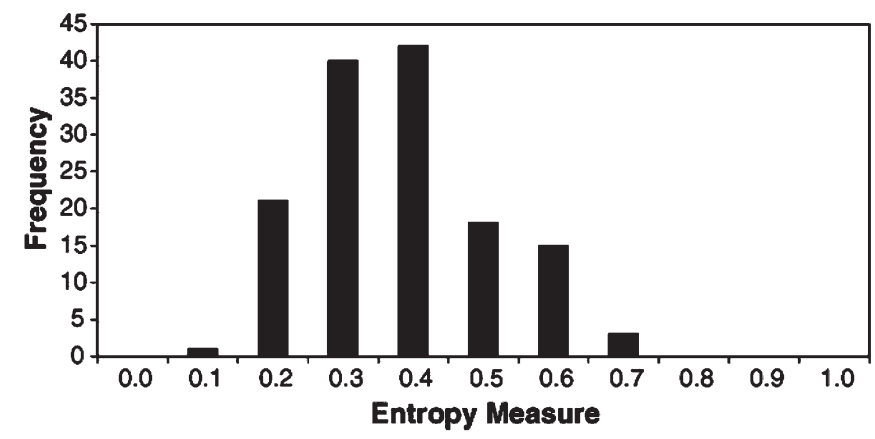

Figure 15. Calculated entropy (using Equation (2)) for three input model test data.

three input model had an $R^{2}$ of 0.61 . The correlation between actual and defuzzified predicted values for the three input model is shown in Figure 13. The three input model had an $R^{2}$ of 0.45 . The correlation between actual and defuzzified predicted values for the two input model is shown in Figure 14. Based on $R^{2}$ values, the three input model has a better performance in predicting crisp $\log K_{p}$ values. In both models, the performance appears to be better at compounds with higher permeability values.

\subsection{Ambiguity for each prediction}

The entropy measure (2) was used to quantify the ambiguity or confidence associated with each test prediction in both models. Figure 15 shows the distribution of this measured entropy for all test cases in the three input model.

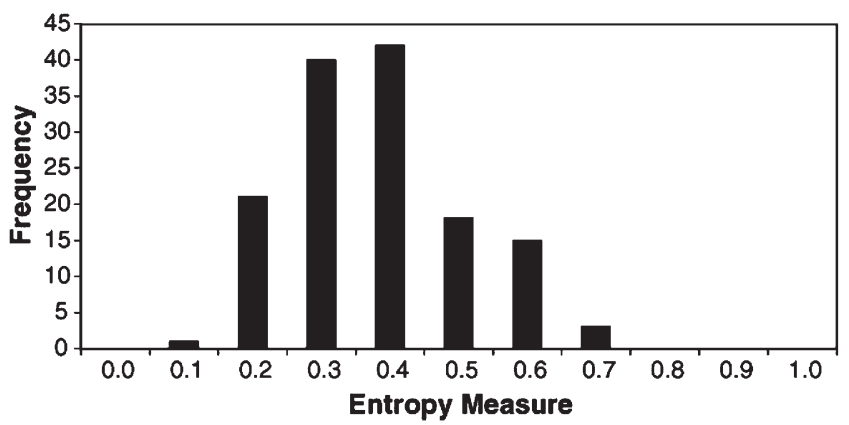

Figure 16. Calculated entropy (using Equation (2)) for two input model test data. 


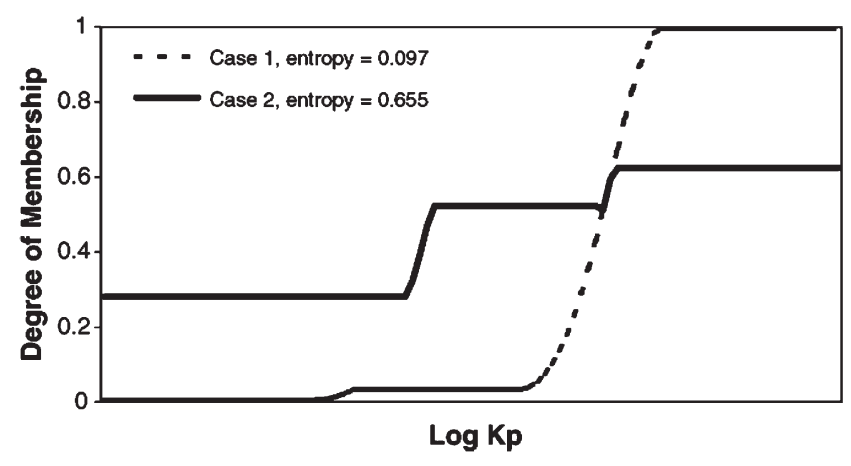

Figure 17. Comparing entropy for predicted fuzzy outputs of two test cases.

Figure 16 shows the distribution of entropy values for test cases used in the two input model. Figure 17 compares the predicted fuzzy output from two sample test cases. Case 1 had a calculated entropy of 0.097 , and case 2 had a calculated entropy of 0.655 . From the shape of the membership function, there is more confidence in the prediction for case 1 than case 2 . Hence, the calculated entropy measures quantify the ambiguity based on shape assessment.

\section{Discussion}

Analysis of the developed Mamdani models involved comparison of actual and predicted fuzzy classifications, correlation between actual and defuzzified crisp values, and calculating entropy to quantify ambiguity. Table 4 compares the performance of the three input model versus the two input model. In every category, the performance of the three input model was better. The $R^{2}$ value obtained for the three input Mamdani model is comparable to results from data driven models by Keshwani et al. [6] using the test data from the same database. Magnusson et al. [8] developed crisp rule-based models to classify compounds based on skin permeability. While a direct comparison between the models is not feasible, the degree of classification of the Mamdani models developed in this study is comparable to results presented by Magnusson et al. [8]. The key difference is that the predictions in the models presented by Magnusson et al. [8] were crisp and not fuzzy as is the case in this study. Taking a fuzzy approach enables the representation of ambiguity associated with each prediction.

The $R^{2}$ values obtained for the models are less than results from some previously published models $[6,7,9,11,13,14]$. However, most previously published models were entirely data driven and optimized for a specific data set. The Mamdani-type model developed is not optimized for a specific data set, and hence it is reasonable to obtain a lower $R^{2}$ value. With more thorough knowledge acquisition and selection of the most significant inputs, the Mamdani-type model will have a better performance. Using multiple knowledge sources and moving away from fitted models can yield a more generalized model for future predictions with new data.

The entropy measures calculated describe the ambiguity associated with the fuzzy prediction. From Figure 17, it
Table 4. Comparison of performance of three input and two input models

\begin{tabular}{lllllr}
\hline & & & $\begin{array}{l}\text { Mean } \\
\text { distance } \\
\text { of test } \\
\text { data }\end{array}$ & $\begin{array}{l}\text { \% of test } \\
\text { data within } \\
\text { half fuzzy } \\
\text { class }\end{array}$ & $\begin{array}{l}\text { Mean } \\
\text { entropy }\end{array}$ \\
\hline Three input & $\log K_{\mathrm{ow}^{\prime}} M_{\mathrm{W}^{\prime}} T$ & 0.61 & 0.32 & 71 & 0.41 \\
Two input & $\log K_{\mathrm{ow}^{\prime}} M_{\mathrm{W}}$ & 0.45 & 0.38 & 47 & 0.49 \\
\hline
\end{tabular}

is clear that the prediction for case 1 is much better than case 2 . For future predictions, when the actual value is not known, the entropy measure provides an estimate of confidence for the prediction (fuzzy or defuzzified). Data-driven models provide a crisp estimate for future predictions. But other than referring to past performance with test data, there is no clear estimate on how good the prediction is for the new data point. Using entropy measures to quantify ambiguity addresses this issue.

\section{Conclusion}

Two Mamdani-type models were developed to predict skin permeability coefficients using octanol-water partition coefficient, molecular weight, and temperature as inputs. Using multiple knowledge sources, membership functions and rules were developed to provide generalized models not optimized for a specific data set.

Apart from correlation estimates of actual and defuzzified predictions, an alternative analysis was performed involving comparison of actual and predicted fuzzy classifications. A distance measure was used to compare actual and fuzzy classifications. The proposed measure is a modification of the Hamming distance often used to compare distances between fuzzy sets. One of the drawbacks of the proposed distance measure is that it does not take into account the direction of misclassification. The entropy measure used also appears to have a drawback: it does not clearly distinguish between unimodal and slightly bimodal fuzzy outputs.

The Mamdani model developed is a knowledge-driven predictive model that is not common in skin permeability literature. A major advantage of this modeling approach is that it enables the use of entropy measures to quantify ambiguity associated with future predictions. This provides a measure of confidence for predicting $\log K_{p}$ for compounds when the actual value is unknown. Potential uses of the presented models include rapid assessment of skin permeability of compounds to identify candidates for transdermal drug delivery and estimate toxicity risks.

\section{References}

[1] R. Babuska, Fuzzy Modeling for Control, Kluwer Academic Publishers, Massachusetts, 1998.

[2] D. A. Edwards, R. Langer, A linear theory of transdermal transport phenomena, J. Pharm. Sci. 83 (1994) 1315-1334.

[3] G. L. Flynn, Physiochemical determinants of skin absorp- 
tion, in: T. R. Gerrity, C. J. Henry (Eds.), Principles of Routeto-Route Extrapolation for Risk Assessment, Elsevier, New York, 1990, pp. 673-715.

[4] W. Hung, A note on entropy of intuitionistic fuzzy sets, Int. J. Uncert. Fuzz. Knowledge-Based Syst. 11 (2003) 627-633.

[6] D. R. Keshwani, D. D. Jones, R. M. Brand, Takagi-Sugeno fuzzy modeling of skin permeability, Cutan. Ocular Toxicol. 24 (2005) 149-163.

[7] L. A. Kirchner, R. P. Moody, E. Doyle, R. Bose, J. Jeffrey, I. $\mathrm{Chu}$, The prediction of skin permeability by using physicochemical data, Altern. Lab. Anim. 25 (1997) 359-370.

[8] B. M. Magnusson, W. J. Pugh, M. S. Roberts, Simple rules defining the potential of compounds for transdermal delivery or toxicity, Pharm. Res. 21 (2004) 1047-1054.

[9] R. P. Moody, H. MacPherson, Determination of dermal absorption QSAR/QSPRs by brute force regression: multiparameter model development using MOLSUITE, 2000, J. Toxicol. Environ. Health-Part A 66 (2003) 1927-1942.

[10] V. Oduguwa, R. Roy, D. Farrugia, Development of a soft computing-based framework for engineering design optimization with quantitative and qualitative search spaces, Appl. Soft Comput. 7 (2007) 166-188.
[11] A. K. Pannier, R. M. Brand, D. D. Jones, Fuzzy modeling of skin permeability coefficients, Pharm. Res. 20 (2003) 143-148.

[12] D. T. Pham, M. Castellani, Action aggregation and defuzzification in Mamdani-type fuzzy systems, in: Proceedings of the Institute of Mechanical Engineers Part C, J. Mech. Eng. Sci. 216 (2002) 747- 759.

[13] R. O. Potts, R. H. Guy, Predicting skin permeability, Pharm. Res. 9 (1992) 663-669.

[14] R. O. Potts, R. H. Guy, A predictive algorithm for skin permeability - the effects of molecular size and hydrogen bonding activity, Pharm. Res. 12 (1995) 1628-1633.

[15] T. J. Ross, Fuzzy Logic with Engineering Applications, McGraw-Hill Inc., New York, 1995.

[16] E. Szmidt, J. Kacpryzk, Distances between intuitionistic fuzzy sets, Fuzzy Sets Syst. 114 (2000) 505-518.

[17] B. E. Vecchia, A. L. Bunge, Skin absorption databases and predictive equations, in: J. Hadgraft, R. H. Guy (Eds.), Transdermal Drug Delivery Systems, vol. 123, Marcel Dekker, New York, 2003, pp. 57-141. 